\title{
Oil Palm Leaf: A New Functional Food Ingredient for Health and Disease Prevention
}

\section{Suhaila Mohamed*}

Institute of Bioscience, University Putra Malaysia 43400 Serdang Selangor, Malaysia

\begin{abstract}
The Oil palm (Elaeis quineensis) leaves has $8 \%$ higher total polyphenols content than green tea extract and contains epigallocatechin, catechin, epicatechin, epigallocatechin gallate, epicatechin gallate amongst their various flavonoids glucosides. The health benefits of OPLE on breast cancer, diabetes, hypertension, inflammation, oxidative stress, neurodegeneration, cognitive functions, dyslipidemia, cardiovascular, liver, kidney and neuroprotective properties and hypercholesterol are reviewed. The OPLE have good vasodilative, phytoestrogenic and bone mass enhancing properties, valuable for postmenopausal women. The OPLE helped modulate the antioxidative defence enzymes and reduce blood Low Density Lipoprotein (LDL) by up-regulating the LDL receptors ex vivo. The safety and acute toxicity of OPLE showed a no adverse effect dose at $2 \mathrm{~g} / \mathrm{kg}$. Sub-chronic daily supplement of $500 \mathrm{mg} / \mathrm{kg}$ body weight (bw) for 3 months in rodents showed no significant adverse effects. The catechin-rich OPLE on its own at $500 \mathrm{mg} / \mathrm{kg}$ body weight/day was not toxic to normal rodents under subchronic continuous consumption for 3 months and was shown to be safe for humans at $1 \mathrm{~g} /$ day for prolonged periods.
\end{abstract}

Keywords: Oil palm leaf; Extract; Health; Polar extract; Human; Animal

\section{Introduction}

The oil palm leaves are a by-product of the palm oil industry and have been used for decades as ruminant feed without any report of toxicity. The palm oil industry is very important to many tropical countries, worldwide to provide a relatively good value and stable food product. In South East Asia, it is common for the palm leaves to be woven into little bags for boiling rice, and the polyphenols from these leaves diffuse into the boiled rice to give it a pleasant flavour and aroma. The main problem for its use as food in its natural form is its high content of insoluble fibre. In 1997 a Japanese patent no. 9206043 filed an application for the preparation and method to produce hemp palm extract which is allegedly beneficial for health. Oil palm (Elaeis guineensis) leaves polar extract (OPLE) or extract has $8 \%$ higher contents of nontoxic, ant oxidative phenolic compounds than green tea extract (mainly common plant constituents like glycosylated flavonoid) [1]. With today's technology of producing polar extract rather competitively, the oil palm leaf may be a potential new source of functional food ingredient, based on various reports of its benefits on health.

\section{Compounds identified in the ople}

The HPLC analysis showed the OPLE contains the main flavonoids as epigallocatechin $\left(800 \mathrm{mg} \cdot \mathrm{kg}^{-1}\right)$, catechin $\left(3 \mathrm{~g} \cdot \mathrm{kg}^{-1}\right)$, epicatechin $(100$ mg. $\left.\mathrm{kg}^{-1}\right)$, epigallocatechin gallate $\left(2.8 \mathrm{~g}^{-\mathrm{kg}^{-1}}\right)$, epicatechin gallate $(500$ mg. $\mathrm{kg}^{-1}$ ) and their glucosides [2]. The OPLE contains vitamin E [3] catechins and other flavonoids such as ferulic acid, chlorogenic acids and other bio-phenolic compounds (gallic acid, protocatechuic acid) [2] that were potently antioxidative and phytoestrogenic [4]. The beneficial effects of OPLE are probably caused by the synergistic effects of these compounds with the catechins.

\section{Antioxidant properties (In vitro, ex vivo and in vivo)}

The OPLE effectively inhibited $\mathrm{Cu}^{2+}$-mediated Low Density Lipoprotein (LDL) oxidation ex vivo [5]. LDL oxidation accelerates atherosclerosis development and dietary antioxidants are protective against the associated cardiovascular disorders. Oxidized LDL are atherogenic because they (1) tend to enter into and accumulate in the arterial wall causing endothelial damage; (2) can form various biologically active molecules (such as oxidized sterols, oxidized fatty acid and protein derivatives) which diversely effect the arterial wall related to atherosclerosis; and (3) is not recognized by the LDLreceptor, hence is taken up instead by the scavenger receptor on the monocytes-macrophages causing foam cell formation.

The OPLE antioxidative effects in vivo in whole animal models are evidenced by (1) the reduced tissue MDA levels in high fat high cholesterol (HFHC) fed rats and the modulation of antioxidative defense enzymes (SOD, CAT and GSH-Px) activities [6], (2) decreased the erythrocyte lipid peroxidation and increased GSH and CAT activities in the tumour-induced rodents [4], and (3) downregulation of all the antioxidant enzymes (SOD, GPx and Catalase) without causing significant increases in MDA levels in hypertensive rodents [2]. It indicated that with the OPLE consumption, there was decreased level of oxidative stress, stimulating a decreased need in the first line of endogenous antioxidant defence, and less antioxidant enzymes activities were required to keep the MDA level at a normal physiological value [2]

\section{Preventive and Therapeutic Properties against Degenerative Disease}

\section{Nerves and brain}

Severe oxidative stress can cause functional impairments such

*Corresponding author: Suhaila Mohamed, Institute of Bioscience, University Putra Malaysia 43400 Serdang Selangor, Malaysia, Tel: +6-03-89472168; Fax: +6-0389472101; E mail: mohamed.suhaila@gmail.com

Received January 15, 2014; Accepted February 18, 2014; Published February 28,2014

Citation: Mohamed S (2014) Oil Palm Leaf: A New Functional Food Ingredient for Health and Disease Prevention. J Food Process Technol 5: 300. doi:10.4172/21577110.1000300

Copyright: (c) 2014 Mohamed S. This is an open-access article distributed under the terms of the Creative Commons Attribution License, which permits unrestricted use, distribution, and reproduction in any medium, provided the original author and source are credited. 
as cognitive decline, due to cellular enzymes deterioration, and consequently exacerbates the neurodegenerative process. Peroxynitrite and other free radicals have been blamed for neurological disorders, chemical sensitivity, inflammation, fibromyalgia, chronic fatigue syndrome, post-traumatic stress disorder, and many disease conditions. A reduction in hippocampal lipid peroxides (LPO) improved spatial cognition and learning memory in aged rodents [7]. Oxidative stressinduced neuronal apoptosis can be prevented by polyphenols and EGCG on neuronal cells [8,9]. Elevated hippocampus antioxidative activity prevents and ameliorates learning ability impairment in animals. Dietary components affect cognitive performance because the CNS relies heavily on a constant supply of essential nutrients and oxygen via the blood supply, for efficient functioning. The OPLE consumption ( $0.5 \mathrm{~g}$ water extract/day equivalent to $2-3$ cups of extract) by young, healthy, adult human volunteers, significantly improved their cognitive learning abilities when compared to the control group $(\mathrm{N}=15)$. Subjects taking OPLE had improved $(\mathrm{p}<0.05)$ short-term memory, after one month of intervention which became highly significant $(\mathrm{p}<0.005)$ after two months, compared to the control subjects. The spatial visualization ability and processing speed were improved $(\mathrm{p}<0.05)$ after two months consumption compared to the control group and the baseline [10]. The dietary OPLE showed neuroprotection and enhanced neuron survival in NO-deficient animals. The OPLE modulated the brain antioxidant enzymes in NO-deficient animals, and indicated neurogenesis in normal control animals. Results supported the cognitive enhancing effects seen in humans.

\section{Obesity and dyslipidemia prevention and therapy}

Prolonged consumption of high fat high cholesterol (HFHC) diet causes significant increases in body weight (BW), plasma total cholesterol (TC), plasma low-density-lipoprotein-cholesterol (LDL-C), plasma atherogenic index (AIP), and lipid peroxidation in various organs compared to normal diet $(\mathrm{N})$. The OPLE consumption with HFHC diet significantly retards these increases in rats. The OPLE also increased the good high-density-lipoprotein-cholesterol (HDL-C) compared to un supplemented HFHC rats [6].

\section{Mechanisms for anti-dyslipidemic effects}

Catechins with a galloyl moiety, suppresses postprandial hyper triacyl glycerolemia by inhibiting pancreatic lipase, triglyceride absorption, delaying lymphatic transport of dietary fat [11]. The catechins lower intestinal cholesterol absorption by inhibiting cholesterol micellar solubility. Catechins suppress visceral fat deposition in animals and humans, without accelerating $\beta$-oxidation in rats' liver [11]. The flavonoid inhibits 3-hydroxy-3-methylglutaryl coenzyme A (HMG$\mathrm{CoA}$ ) reductase (the rate-limiting enzyme in cholesterol synthesis) by (1) inhibiting cAMP PDE, which cleaves coenzyme cAMP [12], causing cAMP to increase and enhance HMG-CoA reductase phosphorylation to the inactive form, thus reducing endogenous cholesterol production, (2) retarding the liberation of the aliphatic phosphoesters from HMGCoA reductase by attaching to the $\mathrm{Zn}^{2+}$ in the protein phosphatase active site, to retain its inactivity [13].

Reduced liver cholesterol synthesis and intracellular cholesterol levels by the polyphenols, will consequently cause hepatocytes membrane LDL receptors up-regulation. This enhances apolipoprotein E- and B-containing lipoproteins removal from the circulation, reduces the synthesis and secretion of lipoproteins from the liver, boosts the uptake of non-HDL particles, and the removal of LDL, IntermediateDensity Lipoprotein (IDL) and VLDL remnant particles from the circulation. Bile acid sequestrants inhibit bile acid reabsorption in the ileum, increasing fecal bile acid loss, causing more up regulation of both bile synthesis (hepatic cholesterol breakdown) and hepatic LDL receptors. Bile acid synthesis is enhanced by increased LDL influx into the liver and further promotes plasma LDL-C decreases [14].

Catechins increased LDL receptor binding activity, protein, and mRNA at the gene transcription level in human HepG2 liver cells [15]. Catechins decreased cell cholesterol and increased the conversion of sterol-regulated element binding protein (SREBP-1) from the inactive precursor to the active transcription-factor form. Catechins elevates HMG-CoA reductase mRNA and up-regulates the LDL receptor in HepG2 cells, partly through SREBP-1 when intracellular cholesterol concentration decreases [15]. Catechins reduced LDL oxidation, macrophages LDL uptake, LDL aggregation and atheromatous lesions in apoE-deficient mice [16], besides inhibiting smooth muscle cells invasion and proliferation in the arterial wall [17].

The OPLE help increase the beneficial HDL-C that translocate cholesterol from the peripheral tissue such as arterial walls to the liver for catabolism and excretion in the bile. High HDL-C protects against atherogenesis and inhibits LDL oxidation [18]. Regular catechins and procyanidins consumptions increased HDL-C levels and reduced LDL oxidative susceptibility in humans [19]. The OPLE decreases the Atherogenic Index of Plasma (AIP) in HFHC-fed rats, indicating its protective properties against atherosclerosis and CVD risk. AIP expressed as $\mathrm{LOG}_{10}$ (TG/HDL-C), correlates with the size of pro- and anti-atherogenic lipoprotein particles, and reflects the balance between the atherogenic and protective lipoproteins. The statin drugs will generally lower LDL-C level, but they will not increase the HDL-C level, and statins can cause muscular weakness with prolonged use.

The Whitehall II study reported that low HDL-C enhances midlife memory decline risks [20]. HDL-C is needed for a healthy brain function. Low HDL-C and elevated TC, LDL-C increases the expression of Alzheimer's disease (AD)-related pathology [21]. AD patients had significantly lower HDL-C and very significantly higher LDL-C, ApoB, A $\beta$ N-40 and A $\beta$ N-42 than normal. TC, LDL-C, and ApoB were positively correlated to $\mathrm{A} \beta \mathrm{N}-42$ levels in $\mathrm{AD}$ brains, which were independent of ApoE genotype. OPLE consumption lowered LDL-C while raising the plasma HDL-C levels in animals and humans, and these data are in agreement with the cognition studies.

\section{Diabetes}

Diabetes mellitus is expected to reach $10 \%$ of the adult population worldwide. The OPLE effectively reduced blood glucose and lipid oxidation in Type II diabetic humans and diabetes-induced rodents. The optimum dose in animal studies is equivalent to consuming 5 cups of $1 \%$ palm leaf extract for diabetic humans, to prevent liver and kidney damage. The OPLE restored diabetic rats' antioxidative defence response enzymes to normal levels and modulated various physiological responses to protect against tissue damages [22].

\section{Hypertension}

Hypertension is an important risk factor for stroke and tissue or organ damage. The OPLE inhibited blood pressure, lipid peroxidation and coronary arteriole wall-lumen ratio increases in vivo, via elevating serum NO and antioxidant effects in NO-deficient hypertensive rats [2]. Although OPLE showed good antihypertensive and antioxidant effects in NO-deficient rats, it was not hypotensive to normal rats, and produced no significant toxicity in the 3 months subchronic 
feeding study. Various case-control and cohort studies showed extract or catechin consumption is epidemiologically associated to lower myocardial infarction risk [23] and lower coronary death risk but not to stroke [24]. Tea polyphenols have endothelial-dependent vasorelaxing activity and retard endothelial dysfunction (an atherosclerosis risk factor before plaque formation) in humans [25]. Ex vivo studies showed the OPLE can produce vasodilation in noradrenaline preconstricted rat aorta and mesenteric arterial bed arteries, via mainly endothelium dependent mechanisms [26]. The OPLE has also been shown to maintain Arterial Homeostasis (Malaysian patent application PI2011005992).

\section{Anti-tumor, anticancer and antiproliferative properties}

The OPLE when given to breast tumor-bearing rodents showed both preventive and therapeutic tumour suppressive effects [4]. Rat mammary tumor was induced with subcutaneously injected LA-7 cells. Hormonal levels, oxidative status, tumour incidence rate and tumour size monitoring showed the OPLE kerbed tumour incidence and volume in a dose and time dependent manner. The phytoestrogenic OPLE enhanced apoptosis, serum estradiol levels and antioxidative status of the tumour-bearing rodents [4].

\section{Phytoestrogenic properties}

The OPLE caused significant 2.54-fold increase in circulating $17 \beta$-estradiol levels, vaginal cornification and uterine wet weight in estrogen-deficient ovariectomized rats, when compared to the un-supplemented control and the effects were dose dependent. The OPLE lengthened the estrus (fertile) phase of the estrous cycle in a dose dependent manner. Restoration of normal estrous cycles after withdrawal of OPLE showed that the effect was reversible in normal animals. The estrogenic activity shown by OPLE can be attributed to the presence of flavonoids and phenolic compounds [4]. Flavonoids have certain similarity in structure and function to estrogen. This estrogenic property of OPLE may be a possible explanation for the OPLE effects on the blood lipid profile. Estrogen receptor alpha (ER- $\alpha$ ) and estrogen related receptor alpha (ERR- $\alpha$ ) regulate various similar genes including lactoferrin, osteopontin, Medium-Chain Acyl Coenzyme a Dehydrogenase (MCAD), thyroid hormone receptor genes and genes involved in mitochondrial biogenesis, gluconeogenesis, oxidative phosphorylation and fatty acid metabolism. ERR- $\alpha$ is most highly expressed in tissues that preferentially use fatty acids as energy sources such as kidney, heart, cerebellum, intestine, and skeletal muscle, although ERR- $\alpha$ is widely distributed in other tissues. The protein encoded by ERR- $\alpha$ is a nuclear receptor that is closely related to the estrogen receptor, and acts as a site-specific transcription regulator shown to interact with estrogen and the transcription factor TFIIB by direct protein-protein contact. The ERR- $\alpha$ appears to modulate the activity of ER- $\alpha$ in various tissues including breast, uterus, and bone. The isoflavone phytoestrogens genistein and daidzein are non-selective ERR- $\alpha$ agonists [12].

\section{Organ and Tissue Regeneration, Repair and Protection}

\section{Liver}

The OPLE consumption reduced abnormal hepatocytes in hypertensive and/or NO-deficient rodents [2] and reduced hepatocytes swelling, vacuolation and other hepatocellular injury to near normal conditions in hypertensive rodents. The dose reported is equivalent to 2.5 cups of extract or $0.5 \mathrm{~g}$ catechins/day for humans. The liver and kidney detoxifies and metabolizes endogenous and exogenous chemicals, making them vulnerable to injury. Hypertension and NOdeficiency eventually causes vacuolations in the liver, hepatocytes necrosis and injuries. In the hepatic microcirculation, $90 \%$ of the blood enters through the portal vein, and/or resistance changes occur in the hepatic sinusoid micro vascular network. Disruption of liver tissue architecture and vacuolation takes place during hypertension and/or NO-deficiency, together with hepatic fatty infiltration and hepatocellular injury [27]. The NO help maintain hepatic microvasculature and local NO-deficiency contribute to local micro vascular perfusion failure, portal hypertension, hepatic resistance increase, and mimicked other vascular dysfunctions like arteriosclerosis. One way, in which OPLE help protect the liver from damage under NO-deficiency, is through their BP lowering properties. The OPLE increases Endothelium Dependent Hyperpolarizing Factor (EDHF) and reduces the portal pressure under NO-deficiency [2]. The endothelium controls vasomotor tone, vascular homeostasis, and inflammatory processes by producing various compounds under different physical and chemical stimuli [28]. Endothelial dysfunction is indicated by decreased vascular NO level in endothelial cells, among others. Although lower BP would improve hepatic micro vascular perfusion and protect the hepatocytes from damage, captopril which effectively normalized BP, could not protect against damages caused by NO-deficiency. The OPLE consumption could not reduce the BP in spontaneously hypertensive rodents, yet still showed hepatoprotective effects towards them. Hepatic injury are linked to dyslipidemia, lowgrade inflammation and serum NO linked insulin resistance. Unlike the hypertensive drug Captopril, which may cause liver injury, the OPLE showed no observable injury, chronic hepatotoxicity or toxicity to normal animals [29]. Enhanced lipid peroxidation in different tissues, result in a range of toxic aldehydes that are capable of damaging nucleic materials, proteins and membranes [30], leading to the leakage of cytoplasmic enzymes [31].

\section{Kidneys}

The OPLE consumption can reduce glomeruli damage caused by chronic hypertension [29]. The Malondialdehyde (MDA-lipid peroxide biomarkers), blood urea nitrogen (BUN) and plasma creatinine levels were significantly reduced in NO-deficient rats supplemented with OPLE to normal rat levels, also indicating the OPLE antioxidative properties [2]. Elevations in plasma BUN or creatinine levels occur when more than $70 \%$ of the nephrons are non-functional. Chronic NO-deficiency caused significant elevation in BUN levels and OPLE consumption reduced BUN level to normal. The OPLE retard lipid peroxidation and protect against NO-deficiency mediated lipid peroxidation. The catechins-rich OPLE also helped reduce kidney injury, dyslipidemia and atherogenicity in high-fat-high-cholesterol diet rodents by modulating their defence enzymes [6].

\section{Heart and coronary small arteries}

The OPLE consumption significantly reduced MDA and SOD activity in NO-deficient rodents' hearts when compared with control and NO-deficient hypertensive rodents [2]. There were decreases in artery wall thickness and wall to lumen ratios to normal values with OPLE consumption. The coronary artery walls were noticeably thickened after 3 months of NO deficiency with significant changes in the wall to lumen ratio when compared to the normal rodents.

\section{Bone}

Estrogen deficiency such as after menopause or ovariectomy 
often causes bone-density loss and osteoporosis. The catechins-rich OPLE dose dependently increased estrogen-deficient bone-density / bone mass loss and structure, bone calcium loss and blood T-ALP concentrations by up to $76 \%$ depending on the parameters monitored. The OPLE enhanced bone mass in estrogen deficient rodents by increasing osteoblast activities to higher levels than in normal rodents and green tea extract, which is evidenced by the modulation of serumT-ALP levels, bone calcium content, total mineral content and bone histological structure. The OPLE is a potential inexpensive extract for protection against osteoporosis and influencing bone metabolism by directing towards bone formation and enhancing the activities of osteoblast [32].

\section{Skin: Wound healing and antimicrobial properties}

Effects of palm extract on inflammation and proliferation phases of wound healing (wound contraction, re-epithelization and granulation tissue development) especially in diabetic animals were reported (Malaysian Patent application PI20083894: A Composition For Wound Healing filed on 29-09-2008). It showed that OPLE increased the rate of wound contraction enhanced epithelization and tissue granulation significantly compared with the positive and negative control groups [33]. The oil palm leaves methanolic extract have good antioxidant properties and contains phenolic compounds such as flavonoids, tannins, coumarins, alkaloids, saponins, terpenoids, steroids, and carbohydrates, with flavonoids being the main constituent [34]

\section{Antimicrobial properties}

The E. guineensis leaf extract alcoholic extracts showed broad antibacterial spectrum activity to all tested bacteria with inhibition zone of $7.7-11.3 \pm 1.0 \mathrm{~mm}$ [34], and were especially effective against S. aureus with a Minimum Inhibition Concentration (MIC) of 6.25 $\mathrm{mg} / \mathrm{mL}$ as compared to Chloramphenicol MIC of $30.00 \mu \mathrm{g} / \mathrm{mL}$. Under the SEM and TEM (scanning and transmission electron microscopes) changes observed included cell membrane structural disorganization after $12 \mathrm{~h}$ and total cell collapse $36 \mathrm{~h}$ after exposure to the extract [35]. The extract MIC for other microbes was between $6.25-12.50 \mathrm{mg} / \mathrm{ml}$. It inhibited Candida albicans yeast early growth phase by causing physiological and morphological changes [36].

\section{Immunomodulation/inflammation/allergy}

Most chronic conditions (ageing, cancer, diabetes, cardiovascular disease, allergies, AIDS) are linked to hyper or hypo-active immune functions. The immunoregulatory properties of OPLE were tested in aged rodents. Delayed type hypersensitivity, induced by intraperitoneal injection of sheep red blood cells, was measured by footpad inflammatory response, and used as an indicator of cell mediated immunity. The OPLE showed biphasic effects that enhanced inflammation at low dose with significant late phase oedema inhibition properties while significantly inhibiting inflammation at a higher dose [37]. The OPLE showed potent in-vivo immunoregulatory effects and may be potentially useful for various disorders related to cell mediated immune responses. At the high dose of $300 \mathrm{mg} / \mathrm{kg}$, paw oedema was significantly suppressed within the first 6 hours as compared to the control group. The OPLE at $150 \mathrm{mg} / \mathrm{kg}$ body weight decreased lymphocyte counts, but less severely than dexamethasone. The OPLE possess good biphasic in-vivo immune-regulatory response to injury or inflammation.

\section{Toxicity assessment of OPLE in rodents and humans}

Prolonged consumption at up to $300 \mathrm{mg}$ OPLE/ $\mathrm{kg}$ body weight showed no adverse effects in rodents whatsoever [22]. Humans taking $0.5 \mathrm{~g}$ OPLE/day for 3 months reported very little adverse effects, if any [10]. Diabetic patients taking $1 \mathrm{~g} /$ day of OPLE showed sugar reduction and insulin increases (unpublished).

The rodents given a single dose of either $2 \mathrm{~g} / \mathrm{kg}$ bw showed noobserved adverse effects (NOAE). When given at the very high dose of $5 \mathrm{~g} / \mathrm{kg}$ bw, behavioral changes, grooming, tachycardia, heavy breathing and weakness lasted for 2 hours. The $5 \mathrm{~g}$ dose significantly $(\mathrm{P}<0.05)$ reduced the RBC, hemoglobin and PCV, but not the lymphocytes and WBC. Hepatocyte damage showed recovery by the $14^{\text {th }}$ day. The kidneys showed no changes. There was no death even at the $5 \mathrm{~g}$ dose, and the acute toxicity injuries were reversible. In view of the NOAE dose of $2 \mathrm{~g} / \mathrm{kg}$, the OPLE at the normal dose levels may be considered relatively safe for use [37]. The acute oral toxicity and brine shrimp lethality of a E. guineensis alcoholic extract at $5 \mathrm{~g} / \mathrm{kg}$ was nontoxic and showed no adverse effects (normal behavioral pattern, clinical signs and histology of vital organs) nor mortalities. This was confirmed by toxicity test against brine shrimp whereby the $50 \%$ lethal concentration $\left(\mathrm{LC}_{50}\right)$ values was more than $1.0 \mathrm{mg} / \mathrm{mL}$ ( 9 and $4 \mathrm{mg} / \mathrm{mL}$ at 6 and $24 \mathrm{~h}$, respectively). Both tests confirmed that E. guineensis is nontoxic and safe for commercial utilization.

\section{Cellular mechanisms for health benefits}

Dietary polyphenols help reduce oxidative damage, and the related chronic diseases risk. Many of them interfere with signal transduction regulation at different levels: modulating hormones/growth factors activities, inhibit oncogenes and activate tumour suppressor genes, induce terminal differentiation, activate apoptosis (cancer cell death), restore immune response, inhibit angiogenesis and decrease inflammation. Phenolic compounds reportedly enhance vascular nitric oxide (NO) activities, either by inducing NO production through Nitric Oxide Synthase (NOS) expression or by their direct antioxidant effect, thereby protecting NO against oxidative destruction.

Catechins mitigated organ tissue injury through various reported mechanisms such as (i) antioxidant effects, (ii) central nervous system actions, (iii) gut transport modulations, (iv) fatty acid sequestration and processing, (v) Peroxisome proliferator-activated receptor (PPAR) stimulation, (vi) increasing insulin sensitivity [38], (vii) inflammatory regulation and endothelial apoptosis [39] (viii) enhancing vascular NO production [40], and (ix) preserving vascular homeostasis and endothelial functions [41]. EGCG helped prevent cellular stress by subduing (i) Ang II-overload (ii) reactive oxygen species (ROS) generation and NADPH oxidase over expressions, (iii) NF-kappaB and AP-1 activation, (iv) ROS-dependent $\mathrm{p} 38$ and c-Jun $\mathrm{N}$-terminal kinase (JNK) signaling pathways, (v) EGFR transactivation (vi) extracellular signal-regulated kinase (ERKs) / PI3K / Akt / mTOR /p70(S6K) and (vii) reactivation of Atrial and Brain natriuretic peptide, (markers of chronic heart failure), and (viii) impeding various intracellular signaling transductional pathways [42]. Epicatechin tetramers, epicatechins and its procyanidins oligomers, repressed Acetylcholine esterase (ACE) activity [43]. EGCG but not EGC, supressed Ang IIstimulated (i) Vascular Smooth Muscle Cell (VSMC) hyper-activity, (ii) JNK signaling pathway at transcriptional and posttranslational levels, but seemingly not through ERK and p38 Mitogen-activated protein kinases (MAPK) [44]. Green tea extract catechins also reportedly help prevent brain, liver, and kidney from lipid peroxidation injuries [45].

\section{Summary}

The OPLE has been shown to beneficially affect lipid profile, blood 
pressure, blood physical and biochemical cancer markers and is organ protective. Histological and behavioral observations in human and animal trials to date could not prove any toxic effects of the catechinsrich palm leaf extract with no observable adverse effects (NOAE) at $2 g$ extract/kg body weight. In fact it showed organ protective effects under various pathological conditions. The OPLE are rich in antioxidative catechins and have good cardiovascular as well as phytoestrogenic properties. First phase clinical trials showed the recommended safe efficacious daily dose for humans is in the range of 500-2500 $\mathrm{mg}$ [10]. Exogenous and endogenous antioxidants help decrease cardiovascular disease risk and hypertension. The health benefits of catechins for the prevention of inflammation, oxidative stress, and neurodegeneration, are well documented $[9,46]$. EGCG, the most active component of catechins, acts as an antioxidant in the biological system and was rapidly absorbed and distributed mainly into the mucous membranes of the small intestine and the liver, and can cross the blood brain barrier. Changes in human life style and eating habits resulted in increasing cases of obesity and chronic metabolic diseases (diabetes, heart disease, hypertension, hyperlipidemia and cancer). Many synthetic medicinal compounds have detrimental effects on the liver or kidneys at high doses or prolonged use. OPLE contains beneficial components that are a natural complementary and alternative therapy to drugs to combat these ailing conditions.

\section{References}

1. Runnie I, Nordin MM, Radzali, M, Azizah, H, Hapizah N, et al. (2003) Antioxidant and hypocholesterolemic effects of Elaeis Guineensis leaves extract on hypercholesterolemic rabbits. ASEAN Food J 12:137-147.

2. Jaffri JM, Mohamed S, Rohimi N, Ahmad IN, Noordin MM, et al. (2011) Antihypertensive and cardiovascular effects of catechin-rich oil palm (Elaeis guineensis) leaf extract in nitric oxide-deficient rats. J Med Food 14: 775-783.

3. Kato A, Yamaoka M, Abdul G, Berger KG (1983) Tocopherols of oil palm leaf, JAOCS.

4. Namvar F, Mohamed S, Fard SG, Behravan J, Mustapha NM, et al. (2012) Polyphenol-Rich Seaweed (Eucheuma Cottonii) Extract Suppressed Breast Tumour Via Hormone Modulation \& Apoptosis Induction”, Food Chem 130:376382

5. Salleh MN, Runnie I, Roach PD, Mohamed S, Abeywardena MY (2002) Inhibition of low-density lipoprotein oxidation and up-regulation of low-density lipoprotein receptor in HepG2 cells by tropical plant extracts. J Agric Food Chem 50: 3693-3697.

6. Abdul-Razak M (2009) The effects of Oil Palm Fronds (Elaeis Guineensis) extract on Lipid Profile and Antioxidant Enzyme Activity in Sprague Dawley Rats, MSc Thesis, Universiti Putra Malaysia.

7. Haque AM, Hashimoto M, Katakura M, Tanabe Y, Hara Y, et al. (2006) Longterm administration of green tea catechins improves spatial cognition learning ability in rats. J Nutr 136: 1043-1047.

8. Choi YT, Jung CH, Lee SR, Bae JH, Baek WK, et al. (2001) The green tea polyphenol (-)-epigallocatechin gallate attenuates beta-amyloid-induced neurotoxicity in cultured hippocampal neurons. Life Sci 70: 603-614.

9. Mandel S, Weinreb O, Amit T, Youdim MB (2004) Cell signaling pathways in the neuroprotective actions of the green tea polyphenol (-)-epigallocatechin-3gallate: implications for neurodegenerative diseases. J Neurochem 88: 15551569.

10. Mohamed S, Lee Ming T, Jaffri JM (2013) Cognitive enhancement and neuroprotection by catechin-rich oil palm leaf extract supplement. J Sci Food Agric 93: 819-827.

11. Ikeda I (2008) Multifunctional effects of green tea catechins on prevention of the metabolic syndrome. Asia Pac J Clin Nutr 17 Suppl 1: 273-274.

12. Stein RA, McDonnell DP (2006) Estrogen-related receptor alpha as a therapeutic target in cancer. Endocr Relat Cancer 13 Suppl 1: S25-32.

13. Yamanaka N, Oda O, Nagao S (1997) Green tea catechins such as (-)-epicatechin and (-)-epigallocatechin accelerate Cu2+-induced low density lipoprotein oxidation in propagation phase. FEBS Lett 401: 230-234.
14. Venkatesan N, Devaraj SN, Devaraj H (2003) Increased binding of LDL and VLDL to apo B,E receptors of hepatic plasma membrane of rats treated with Fibernat. Eur J Nutr 42: 262-271.

15. Bursill C, Roach PD, Bottema CD, Pal S (2001) Green tea upregulates the low-density lipoprotein receptor through the sterol-regulated element binding Protein in HepG2 liver cells. J Agric Food Chem 49: 5639-5645.

16. Maeda K, Kuzuya M, Cheng XW, Asai T, Kanda S, et al. (2003) Green tea extract catechins inhibit the cultured smooth muscle cell invasion through the basement barrier. Atherosclerosis 166: 23-30.

17. Rigotti A, Miettinen HE, Krieger M (2003) The role of the high-density lipoprotein receptor SR-BI in the lipid metabolism of endocrine and other tissues. Endocr Rev 24: 357-387.

18. Baba S, Osakabe N, Kato Y, Natsume M, Yasuda A, et al. (2007) Continuous intake of polyphenolic compounds containing cocoa powder reduces LDL oxidative susceptibility and has beneficial effects on plasma HDL-cholesterol concentrations in humans. Am J Clin Nutr 85: 709-717.

19. Singh-Manoux A, Gimeno D, Kivimaki M, Brunner E, Marmot MG (2008) Low HDL cholesterol is a risk factor for deficit and decline in memory in midlife: the Whitehall II study. Arterioscler Thromb Vasc Biol 28: 1556-1562.

20. Kuo YM, Emmerling MR, Bisgaier CL, Essenburg AD, Lampert HC, et al (1998) Elevated low-density lipoprotein in Alzheimer's disease correlates with brain abeta 1-42 levels. Biochem Biophys Res Commun 252: 711-715.

21. Rosalina TRT, Mohamed S, Samaneh GF, Noordin MM, Goh YM, et al. (2011) Polyphenol Rich Oil palm leaves extract reduce hyperglycaemia and lipid oxidation in STZ-rats. Int Food Res J 18: 179-188.

22. Peters U, Poole C, Arab L (2001) Does tea affect cardiovascular disease? A meta-analysis. Am J Epidemiol 154: 495-503.

23. Arts IC, Hollman PC, Feskens EJ, Bueno de Mesquita HB, Kromhout D (2001) Catechin intake might explain the inverse relation between tea consumption and ischemic heart disease: the Zutphen Elderly Study. Am J Clin Nutr 74: 227-232.

24. Duffy SJ, Keaney JF Jr, Holbrook M, Gokce N, Swerdloff PL, et al. (2001) Short- and long-term black tea consumption reverses endothelial dysfunction in patients with coronary artery disease. Circulation 104: 151-156.

25. Abeywardena M, Runnie I, Nizar M, Suhaila M, Head R; Suhaila Momamed (2002) Polyphenol-enriched extract of oil palm fronds (Elaeis guineensis) promotes vascular relaxation via endothelium-dependent mechanisms. Asia Pac J Clin Nutr 11 Suppl 7: S467-472.

26. Hoetzel A, Welle A, Schmidt R, Loop T, Humar M, et al. (2008) Nitric oxidedeficiency regulates hepatic heme oxygenase-1. Nitric Oxide 18: 61-69.

27. Behrendt D, Ganz P (2002) Endothelial function. From vascular biology to clinical applications. Am J Cardiol 90: 40L-48L.

28. Jaffri JM, Mohamed S, Ahmad IN, Mustapha NM, Manap YA, et al. (2011) Effects of Catechin-rich Oil Palm Leaf Extract on normal and hypertensive rats kidney and liver. Food Chem 128: 433-441.

29. Husain K, Scott BR, Reddy SK, Somani SM (2001) Chronic ethanol and nicotine interaction on rat tissue antioxidant defense system. Alcohol 25: 89-97.

30. Bagchi M, Bagchi D, Adickes E, Stohs SJ (1995) Chronic effects of smokeless tobacco extract on rat liver histopathology and production of HSP-90. J Environ Pathol Toxicol Oncol 14: 61-68

31. Bakhsh A, Mustapha NM, Mohamed S (2013) Catechin-rich oil palm leaf extract enhances bone calcium content of estrogen-deficient rats. Nutrition 29 : 667-672.

32. Anyanji VU, Hair BB, Mohamed S (2013) Acute Toxicity Assessment Of Oi Palm (Elaeis Guineensis) Leaf Extract In Rats. J Med Plant Res 7: 1022-1029.

33. Yin NS, Abdullah S, Phin CK (2013) Phytochemical constituents from leaves of Elaeis guineensis and their antioxidant and antimicrobial activities. Int J Pharm Pharmaceut Sci 5: 137-140.

34. Rajoo A, Sasidharan S, Jothy SL, Ramanathan S, Mansor SM (2013) Ultrastructural study of Elaeis guineensis (oil palm) leaf and antimicrobial activity of its methanol extract against staphylococcus aureus. Trop J Pharmaceut Res 12: 419-423.

35. Chong KH, Zuraini Z, Sasidharan S, Devi PVK, Latha LY, et al. (2008) Antimicrobial of Elaeis guineensis leaf. Pharmacologyonline 3:379-386. 
Citation: Mohamed S (2014) Oil Palm Leaf: A New Functional Food Ingredient for Health and Disease Prevention. J Food Process Technol 5: 300. doi:10.4172/2157-7110.1000300

36. Syahmi AR, Vijayarathna S, Sasidharan S, Latha LY, Kwan YP, et al. (2010) Acute oral toxicity and brine shrimp lethality of Elaeis guineensis Jacq., (oil palm leaf) methanol extract. Molecules 15: 8111-8121.

37. Prasain JK, Carlson SH, Wyss JM (2010) Flavonoids and age-related disease: risk, benefits and critical windows. Maturitas 66: 163-171.

38. Curin Y, Andriantsitohaina R (2005) Polyphenols as potential therapeutical agents against cardiovascular diseases. Pharmacol Rep 57 Suppl: 97-107.

39. Galleano M, Oteiza PI, Fraga CG (2009) Cocoa, chocolate, and cardiovascular disease. J Cardiovasc Pharmacol 54: 483-490.

40. Moore RJ, Jackson KG, Minihane AM (2009) Green tea (Camellia sinensis) catechins and vascular function. Br J Nutr 102: 1790-1802.

41. Li HL, Huang Y, Zhang CN, Liu G, Wei YS, et al. (2006) Epigallocathechin-3 gallate inhibits cardiac hypertrophy through blocking reactive oxidative speciesdependent and -independent signal pathways. Free Radic Biol Med 40: 17561775.
42. Ottaviani J, Actis-Goretta L, Villordo JJ, Fraga CG (2006) Procyanidin structure defines the extent and specificity of angiotensin I converting enzyme inhibition. Biochimie 88: 359-365.

43. Zheng Y, Song HJ, Kim CH, Kim HS, Kim EG, et al. (2004) Inhibitory effect of epigallocatechin 3-O-gallate on vascular smooth muscle cell hypertrophy induced by angiotensin II. J Cardiovasc Pharmacol 43: 200-208.

44. Sano M, Takahashi Y, Yoshino K, Shimoi K, Nakamura Y, et al. (1995) Effect of tea (Camellia sinensis L.) on lipid peroxidation in rat liver and kidney: a comparison of green and black tea feeding. Biol Pharm Bull 18: 1006-1008.

45. Khan N, Mukhtar H (2007) Tea polyphenols for health promotion. Life Sci 81: 519-533.

46. Sasidharan S, Logeswaran S, Latha LY (2012) Wound Healing Activity of Elaeis guineensis Leaf Extract Ointment. Int J Mol Sci 13: 336-347. 